\title{
An Efficient and Intelligent Recommender System for Mobile Platform
}

\author{
MUHAMMAD JABBAR*, QAISAR JAVAID**, MUHAMMAD ARIF*, ASIM MUNIR** AND \\ ALI JAVED***
}

RECEIVED ON 21.07.2016 ACCEPTED ON 29.05.2017

\begin{abstract}
Recommender Systems are valuable tools to deal with the problem of overloaded information faced by most of the users in case of making purchase decision to buy any item. Recommender systems are used to provide recommendations in many domains such as movies, books, digital equipment's, etc. The massive collection of available books online presents a great challenge for users to select the relevant books that meet their preferences. Users usually read few pages or contents to decide whether to buy a certain book or not. Recommender systems provide different value addition factors such as similar user ratings, users past history, user profiles, etc. to facilitate the users in terms of providing relevant recommendations according to their preferences. Recommender systems are broadly categorized into content based approach and collaborative filtering approach. Content based or collaborative filtering approaches alone are not sufficient to provide most accurate and relevant recommendations under diverse scenarios. Therefore, hybrid approaches are also designed by combining the features of both the content based and collaborative filtering approaches to provide more relevant recommendations. This paper proposes an efficient hybrid recommendation scheme for mobile platform that includes the traits of content based and collaborative filtering approaches in addition of the context based approach that is included to provide the latest books recommendations to user.Objective and subjective evaluation measures are used to compute the performance of the proposed system. Experimental results are promising and signify the effectiveness of our proposed hybrid scheme in terms of most relevant and latest books recommendations.
\end{abstract}

Key Words: Collaborative Filtering, Context Based Approach, Content Based Method, Hybrid Approach, Recommender System.

\section{INTRODUCTION}

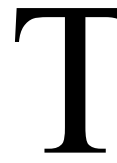

he massive amount of online content availability

creates a problem of overloading of the

a laptop then it can be very hectic experience for him/ her to analyze all available reviews about the laptop of information to the users. If someone has to buy different brands and models before deciding to

Authors E-Mail: (m.jabbar@uog.edu.pk, qaisar@iiu.edu.pk, arifmuhammad36@hotmail.com, asim@iiu.edu.pk, ali.javed@uettaxila.edu.pk) * Department of Computer Science, University of Gujrat, Gujrat.

** Department of Computer Systems \& Software Engineering, International Islamic University, Islamabad.

*** Department of Software Engineering, University of Engineering \& Technology, Taxila

This is an open access article published by Mehran University Research Journal of Engineering and Technology, Jamshoro under the CC by 4.0 International License. 
purchase. Recommender systems are commonly used to address the problem of information overload by recommending most suitable items that meets the interests of the user. Relevant recommendations facilitate the users to easily identify the required items without analyzing any unnecessary information regarding the required product [1]. Recommender systems are also useful for product suppliers to offer the relevant products to their users that satisfy the potential needs of the users in a best possible way. Recommender systems are commonly used to recommend the items of various domains such as movies, news, books, digital products, etc.

Recommender systems are broadly categorized into collaborative filtering based [1] and content based approaches [2]. Collaborative filtering based approaches have further two variations that are neighborhood based collaborative filtering [1] and model based collaborative filtering [1]. Neighborhood based collaborative filtering predicts the rating of an item on the basis of already stored user ratings of other items.Whereas, model based filtering technique utilizes the user ratings to learn predictions. Although collaborative filtering approaches can successfully recommend items based on user preference and item quality, however these approaches have limitations of users rating problem, new user and item problem, sparsity, scalability, and adaptability. Content based filtering approach uses the past history of the users to recommend items. Content based filtering suffers from the problems of limited content analysis, over specialization and new user problems. To address the problems associated with content based and collaborative filtering techniques, hybrid recommendation approaches [3] have also been proposed that integrates the traits of both content based and collaborative techniques. Hybrid approaches combine the useful features of both domains to present more effective recommendations to the users.

Existing mobile based books recommender systems still need many improvements in terms of providing accurate and most relevant recommendation among the books recommendations provided to the user. Moreover, existing systems that are either using collaborative filtering or content based approaches provide limited precision and recall. In most cases the recommendation results of these systems deviate from the potential interests or preferences of the users. Existing research work on books recommendation have applied different techniques to provide the most suitable systems that can provide more relevancy in recommendations. Zhu et. al. [4] designed abook recommendation system using association rule mining. There are some limitations in mining association rule using apriori algorithm. However, efficiency of apriori algorithm is improved through filtering the useful records while ignoring the irrelevant data. This improved algorithm is then used to build a book recommender system using data set from university circulation library records. A service model has also been introduced in [4] to offer the information about the recommendations to users that can also be utilized in other domains i.e. information retrieval, book store service, etc [4]. Rana et. al. [5] proposed a recommender system to provide recommendations about relevant books to the users by finding other users having same preferences. This system [5] introduces a concept of temporal dimension which computes the frequency of user liking of any item in a specific time period. This system provides diverse recommendations in case an item has fewer ratings for specific user.

Mehran University Research Journal of Engineering \& Technology, Volume 37, No. 4, October, 2018 [p-ISSN: 0254-7821, e-ISSN: 2413-7219] 
There exist many book recommender systems in the form of web applications that are used to provide recommendations about different books to the user based on his/her input query. However, all recommendations provided by these systems are not relevant or most relatable to the users' interests or preference and contains much irrelevant information. In addition, we have seen a drastic technology shift in the last decade where we experienced the smart phones revolution. In modern era, every user prefers to use applications on the mobile as compared to desktop/web applications a decade back. Mobile applications are more convenient for users as he/ she can have easy access of application anywhere/ anytime without the need of a big size computer/laptop as mobiles are easy to carry. In addition, mobile phones have constraints of memory and processing which must be considered before development. Therefore, researchers are focusing more towards developing efficient mobile based recommender systems nowadays to meet the needs of the mobile users.

To address the above mentioned limitations of content based and collaborative filtering recommendation approaches we have proposed a hybrid books recommendation approach for mobile platforms. The proposed recommendation scheme is implemented for mobile platform to provide most relevant recommendations to the users based on the users profiles, similar users ratings, and context based scheme of providing the most latest published books. One of the main contribution of the proposed work is to provide the context based scheme in our hybrid recommendation approach besides using the traits of both content based and collaborative filtering approaches. Moreover, the proposed hybrid scheme is developed for mobile platform that provides most relevant and latest books recommendations to the users based on their preference, history, and neighborhood users. In addition, the proposed hybrid scheme uses a simple approach to provide better efficiency to the end users as compared to classifier based approach where the recommendation accuracy can be improved but at the expense of increased computational cost. Performance evaluation of the proposed system is measured in both the objective and subjective way. Precision, recall, accuracy and F-Measure are used for objective evaluation, whereas, usability, relevance, and user satisfaction are used to obtain the subjective ratings of the users. The results indicate the effectiveness of the proposed hybrid book recommendation scheme.

The rest of the paper is organized as follows. Section 2 provides a critical analysis of existing state-of-the-art recommender systems. Section 3 discusses our proposed hybrid recommendation method. Performance evaluation of the proposed method is provided in detail in Section 4. Finally, Section 5 concludes the proposed work and provides future possible recommendations in this domain.

\section{LITERATURE REVIEW}

This section provides a critical analysis of existing stateof-the-art recommender systems. Moreover, a detailed overview of various categories of recommender systems are also discussed in detail. In everyday life, people tend to seek the advice of an expert or ask friends for guidance when they are presented with some unfamiliar choices. Recommender system has been defined in many different ways. Resnick et. al. [6] claims that a customary recommender system aggregates the recommendations provided by the users, as an input, and directs it to an appropriate recipient. A recommender system predicts the items that a user may like based on data from the past [6-7]. A system that generates recommendation as 
output for users or guides them, in a customized way, to find the items that might attract them from a large space of available resources. Factors like individualization and user interest distinguish the recommendation engines from search engines and IR (Information Retrieval) systems [7]. In recent years, online recommender systems are providing the functionality of a social recommendation system. They predict whether a particular user will like a particular product or not. They also identify a set $\mathrm{S}$ of items that will be of interest to a particular user [8].

The exponential growth of the available data content online has motivated the research community to propose effective content management techniques. Recommender systems were initially designed for online shopping and entertainment domain. Nowadays recommender systems are used in every domain such as travel, e-learning, social network, health care, movies recommendations, etc. The availability of an enormous amount of online books content have encouraged the researchers to develop recommender systems for books. There exist several online websites such as amazon, whichbook.net, whatShouldIReadNext.com, libraryThing.com etc. that applies different recommendation schemes to recommend the books to e-user community [9]. Fig. 1 shows the pictorial representation of existing techniques of recommender systems.

The first term coined in the context of recommender ystem is "collaborative filtering". It was developed to generate the list of recommended documents from newsgroups for group of users [10]. Collaborative filtering approach identifies the users that have similar interest with the active user and find the ratings provided by these users to different products, then recommends the list of products to active user that are highly rated by these similar users. Collaborative filtering are further divided into two categories i.e. model based and neighborhood based approach. Neighborhood based approach is commonly

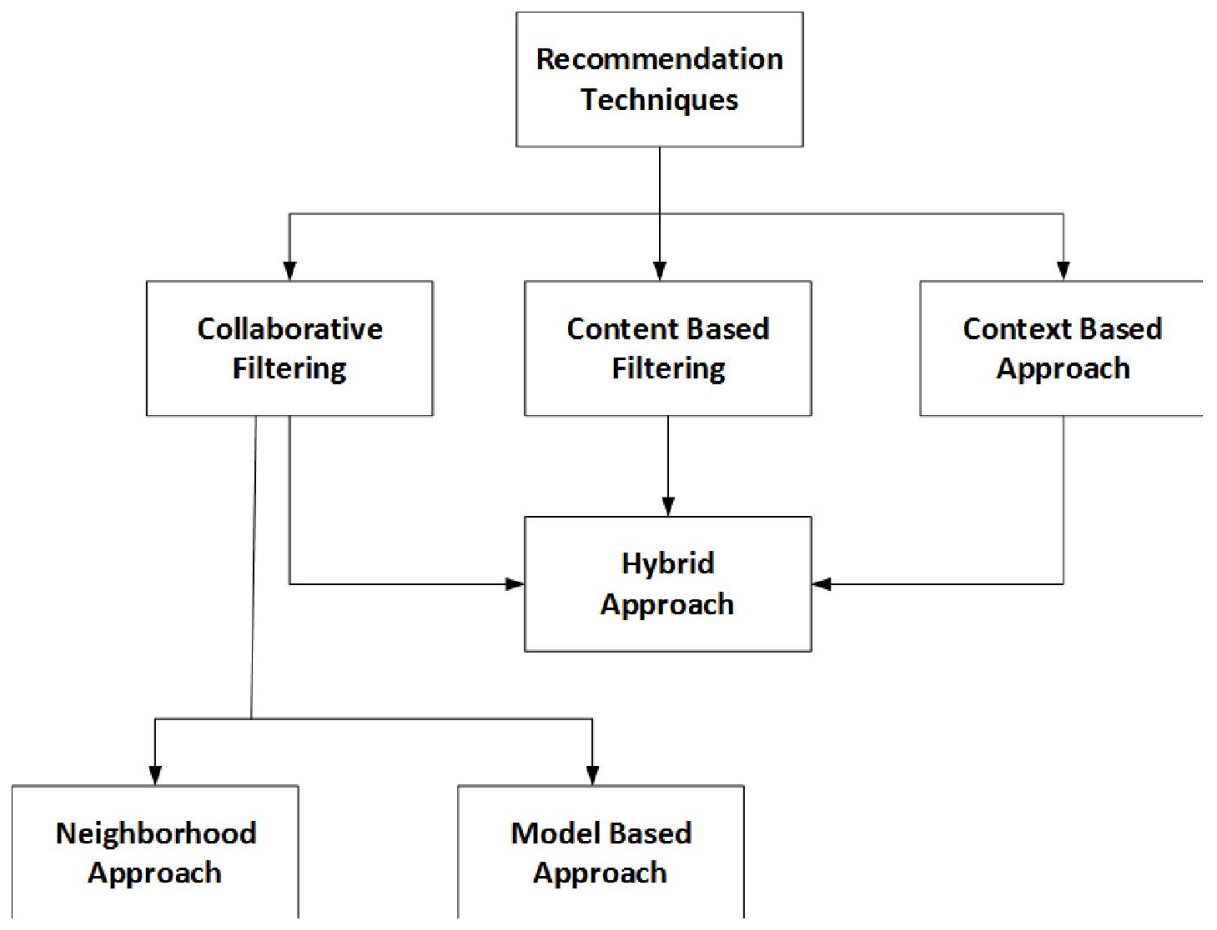

FIG. 1. TECHNIQUES FOR RECOMMENDER SYSTEMS

Mehran University Research Journal of Engineering \& Technology, Volume 37, No. 4, October, 2018 [p-ISSN: 0254-7821, e-ISSN: 2413-7219] 
known as memory based approach [11]. In this approach, a small number of users are selected based on similarity with the active user, then predictions are performed based a weighted mixture of ratings of these users. This approach assigns the weights to all users based on their similarity with the active user. In the next step m number of users are selected having highest similarity to the active user. Finally, prediction is achieved using the weighted ratings of selected users. For similarity computation mechanism it is assumed that unrated products are not the ones with negative or zero ratings [12]. In case of collaborative filtering where we have billions of items and users then neighborhood based approach is not considered effective due to the complexity of finding the similar users. An alternative approach proposed by Sarwar et. al. [13] called item based approach, where user's rated items are matched with similar items instead of finding similar users. It has been claimed in [13] that item based approach lead to quicker online recommender systems and improved recommendation accuracy. In model based approach, recommendations are provided by calculating the statistical model's parameter of user ratings. Matrix factorization and latent factor models are the most commonly used techniques in this approach [14]. Latent factor model considers that likeness between the items and users is persuaded by some unseen lower dimensional data structure. In matrix factorization model, items and users are represented as unfamiliar feature vectors along with latent dimensions.

Content based filtering approaches are also commonly employed besides collaborative filtering based approaches for recommendation. Content based approach uses the past user data to recommend the new item to user as compared to collaborative filtering which provides recommendation based on finding similar users. It uses the user's data such as their preferences, their rating on items, their buying history, their department and then recommends the new item that match with these attributes of user. There exists many research works in this field focusing on recommending the items with textual content for instance books, movies and web-pages. Mooney et. al. [15] have used the data such as title synopses, author, subject items and ratings to train a classifier for books recommendation. The scale of rating is from 1 to $\mathrm{k}$ where mapping is performed among $\mathrm{k}$ different classes. However, different other classification algorithms have also been used purely for content based filtering such as decision tree, K-nearest neighbor and neural network [16].

They presented an argument based hybrid approach for books recommendation. Argumentation facility has been provided to convince the user regarding the utility of the recommended book. In addition, if a user likes more than one book of any author, then the author's popularity index also increases. The author's popularity index is also considered along with other arguments for recommendation. For performance evaluation, recall and precision metrics have been applied.

In recent times context based factors are also included in the conventional recommendation approaches where these factors are combined in either collaborative, content based or hybrid approaches. Context based filtering requires supplementary data about the item's contextual information such as time, behavioral aspects, etc. These context based traits provides a great value addition in conventional recommender systems.

Kim et. al. [17] proposed a hybrid books recommendation approach where a demographics technique has been applied to find similar users. Ontology has been used to store and manage the user's profile. Hashing technique has been used for similar users to avoid the collision. This approach computes average difference among different items and their ratings besides computing the

Mehran University Research Journal of Engineering \& Technology, Volume 37, No. 4, October, 2018 [p-ISSN: 0254-7821, e-ISSN: 2413-7219] 
frequency of rating for each item. Precision, recall and accuracy have been used for performance evaluation. To improve the performance of collaborative and content based filtering, many hybrid approaches have also been proposed that contain the features of both approaches. A simple hybrid approach is to use the collaborative and content based filtering to generate separate recommendation lists followed by integrating them to generate a final list of recommendations [18]. Pera et. al. [19] proposed a personalized recommender system to serve the preferences of wide range of users under diverse conditions. It has been claimed in this paper that existing recommender systems are unable to support the diversity in terms of targeting users of various categories. Therefore, a personalized recommender system was designed based on friendship preferences to meet the diverse user's needs. Ristoski et. al. [20] proposed a multi-strategy hybrid approach that initially combines the output of several base recommenders followed by using generic recommenders to provide the final recommendation. The base recommenders can be either content based or collaborative filtering systems. Generic recommender generates the recommendation based on global popularity marks that are similar for all users. Pathak et. al. [21] uses context based filtering approach along with other techniques to get more precise recommendations. Most of the existing recommender systems are based on context, content and collaborative filtering based approaches. Any of the above mentioned approach alone is not sufficient to recommend the items according to user choice. Therefore, a combination of different approaches can provide better recommendations that can satisfy the user needs in a better way. They proposed a hybrid books recommendation system named "NOVA" to provide more effective recommendations. Smyth et. al. [22] proposed a framework for hybrid approach, where the predictions of content based filtering are used to convert sparse rating matrix to a complete full rating matrix, then collaborative filtering is used for the generation of final recommendation. Existing books recommendation techniques are designed to provide both the online and offline recommendations. Tewari et. al.[23] proposed a books recommender system that provides the recommendations based on user preference. This system is designed to work offline and recommendations are stored in the profile of the buyer. To provide the offline recommendations, outcomes are saved in web profile of buyers. The recommendations produce automatically once the user gets online.

Existing systems also use different mining techniques along with the hybrid approach to identify the user's interests and provide them the books according to their preferences. Sohail et. al. [24] proposed a book recommender system using an opinion mining technique where books are filtered on the basis of several features. Similarly, Sase et. al. [25] have used data mining approach to design a hybrid recommendation engine for books. A short survey is conducted for users to collect the data for demographic filter. Ontology is also used to capture the user preferences for recommendations. DeZelar et. al. [9] used a joint multi-relation technique for recommendation process. Social relationship has been exploited along with other data sources to adjust the latent factors over target matrices. The target matrices include the social relationship and rating of books matrix. Factorization of coupled matrix algorithm has been applied to learn the latent factors. Reader's tag history has also been considered along with the ratings and social relationship. Reader's Tag history is important in the sense that it reflects the book's characters and interest of the reader. According to the reader, books were categorized into read and unread books. The ranking of unread books to a user has been predicted using the explicit rating of books read by the user. Finally, top ranked books were recommended to the user.

Mehran University Research Journal of Engineering \& Technology, Volume 37, No. 4, October, 2018 [p-ISSN: 0254-7821, e-ISSN: 2413-7219] 


\section{PROPOSED SYSTEM}

The proposed system provides a hybrid technique for books recommendation to the users. The reason to adopt a hybrid recommendation technique is to address the limitations of collaborative and content based filtering schemes. The recommendations provided by the collaborative filtering alone does not match the user preferences, whereas, in content based filtering, recommendations are provided on users past buying history thereby ignore the user preferences completely. In the proposed method, content-based and collaborative filtering schemes are merged to develop a hybrid recommender system to provide more effective recommendations to the users. The proposed hybrid approach addresses the limitations of collaborative and content-based filtering by suggesting the books to user according to his/her choice. The recommendations are based on ratings suggested by other users who have similar interest relating to our active user and his/her buying history. An android application is developed based on the proposed hybrid recommendation scheme to evaluate the performance of the proposed method. This section provides a detailed discussion on the system architecture and proposed methodology.

\subsection{System Architecture}

The proposed hybrid approach adopts the collaborative and content based approaches in addition of the context based approach to recommend the top-rated relevant books to the users. The block diagram of the proposed approach is provided in Fig. 2. User can login to our mobile application to view and rate the books and receive the recommendations from our system. Content based filtering is applied through accessing the user profile to obtain the user preferences. Our approach uses the content based filtering to discover the similarity between the active user and other users. Similarly, collaborative filtering is applied to find the similar users that share the same interests and list of books that are rated high by other users. Collaborative filtering scheme obtains the input from the dataset (i.e. rating of books, user's records). This information is processed by the collaborative filtering method to find the similar users and fed this information to our proposed hybrid scheme. The proposed hybrid approach processes the information provided by content based and collaborative filtering techniques to recommend the most relevant books to the users. It also simplifies the querying and retrieving of data and parsing of different log files can be avoided. Moreover, the proposed android application has maintained a record of existing users in the form of user profiles which are used to implement content based approach in our method. In addition, our android application maintains a record of each new user that visits our application for recommendations and create a neighborhood of users with those already stored in the database on the basis of interests' similarity. The similarity measure depends upon various factors such as author, book titles, etc.

The proposed hybrid recommender system is robust against the above mentioned limitations of collaborative filtering and content based approaches. The proposed mobile application offers an easy and interactive interface to users to collaborate with other users by providing the ratings and obtains a recommended list of books for reading purpose. The proposed system successfully provides the relevant recommendations, ease of use, and ultimate user satisfaction to the users. 


\subsection{Proposed Methodology}

This section provides the discussion of the proposed methodology of our hybrid recommendation approach.

Fig. 3 shown the process flow of the proposed recommendation algorithm. The proposed technique is a fusion of collaborative, context based and content based approaches. Here in our case context based method filters the recommended list of books based on the publication year. This context based approach is added in the proposed approach to provide the most recent published books that are included in top-ranked recommended category of books. The flow of the proposed scheme is discussed here.

The proposed system obtains the category of books based on user preferences in the initial step. In step-2, user scanning is performed by the system to identify if the user is visiting the application for the first time or already visited before. If the user already visited the system earlier then the proposed system identifies those books liked or rated high by the user in previous visits. The proposed application then search the database to find the books similar to the ones user liked in step-3. The filtered books are assigned the weights in the next stage as Equation (1):

$\mathrm{J} 1_{\mathrm{i}}+\mathrm{W}_{1} * \mathrm{r}_{\mathrm{ji}}$

Here $r_{\mathrm{ji}}$ is the rating of ith book from list $\mathrm{J}$.

In step-4, the proposed system identifies other users from the user dataset who liked to read the similar category of books which the active user wants to read. After finding the similar users, the system checks the rating of these users from rating table to find their highly rated books. The proposed system then checks the rating's frequency of each book in accordance with the users in step-5. The system then calculates the average dissimilarity between the ratings provided by the active

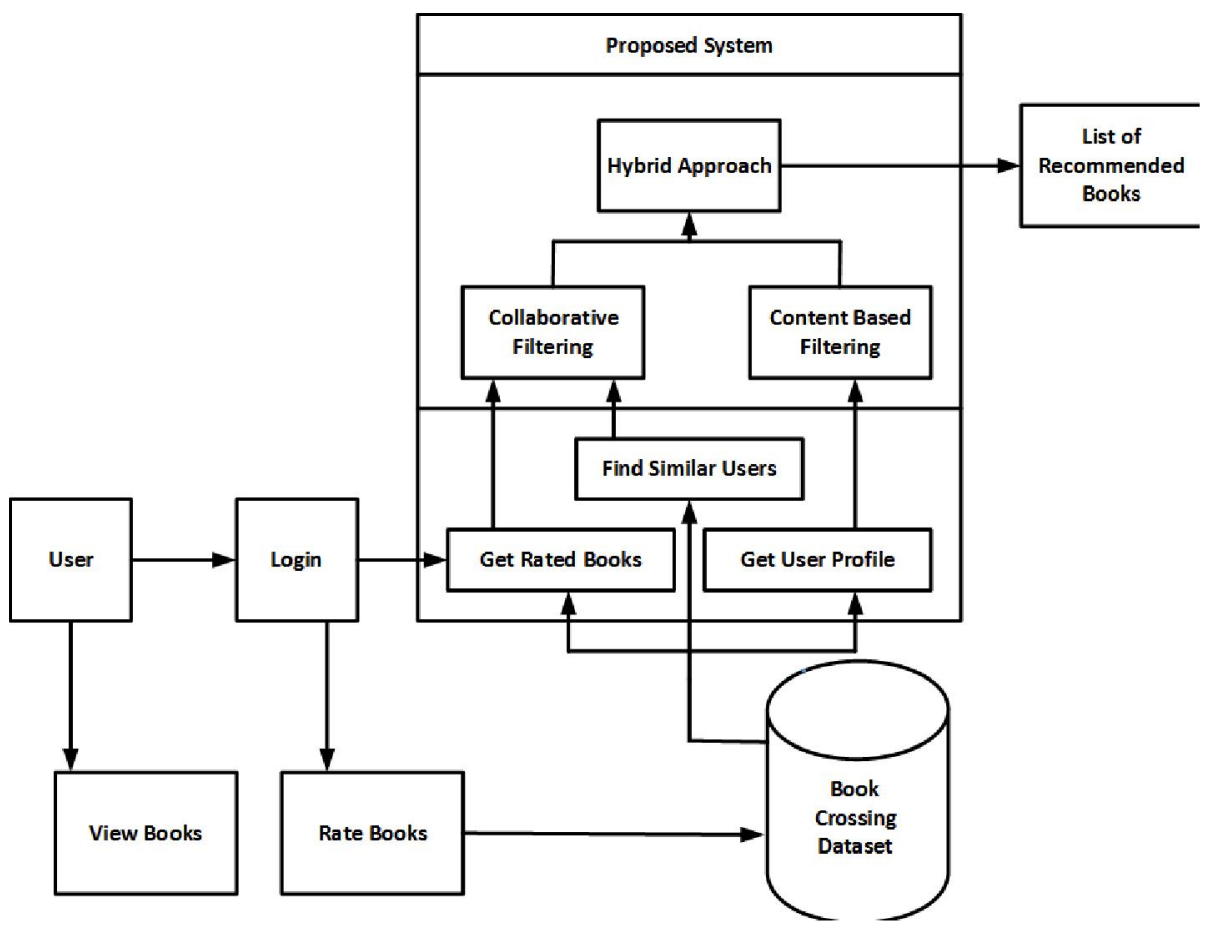

FIG. 2. BLOCK DIAGRAM FOR PROPOSED SYSTEM

Mehran University Research Journal of Engineering \& Technology, Volume 37, No. 4, October, 2018 [p-ISSN: 0254-7821, e-ISSN: 2413-7219] 
user and other users who have similar interest with active user in step-6. The similarity between the users is calculated using Equation (2).

$S(P, P)=\frac{\sum_{i=1}^{m} \sum_{i=1}^{m} S_{c}\left(P c_{i}, P c_{j}\right)}{m^{*} m}$

Where $n$ and $m$ denotes the number of interests provided by user $\mathrm{P}$ and $\mathrm{P}$. And (Pc, 'cj) is the measure of similarity between interests ci and $c_{j}$, and it is computed as Equation (3):

$\mathrm{S}_{\mathrm{c}}\left(\mathrm{Pc}_{\mathrm{i}}, \mathrm{Pc}_{\mathrm{j}}\right)=\log \frac{\mathrm{P}}{\mathrm{Pc}_{\mathrm{j}}}$

Where $\mathrm{P}$ is the total number users and Pci is the number of users whom interest is similar to the active user.

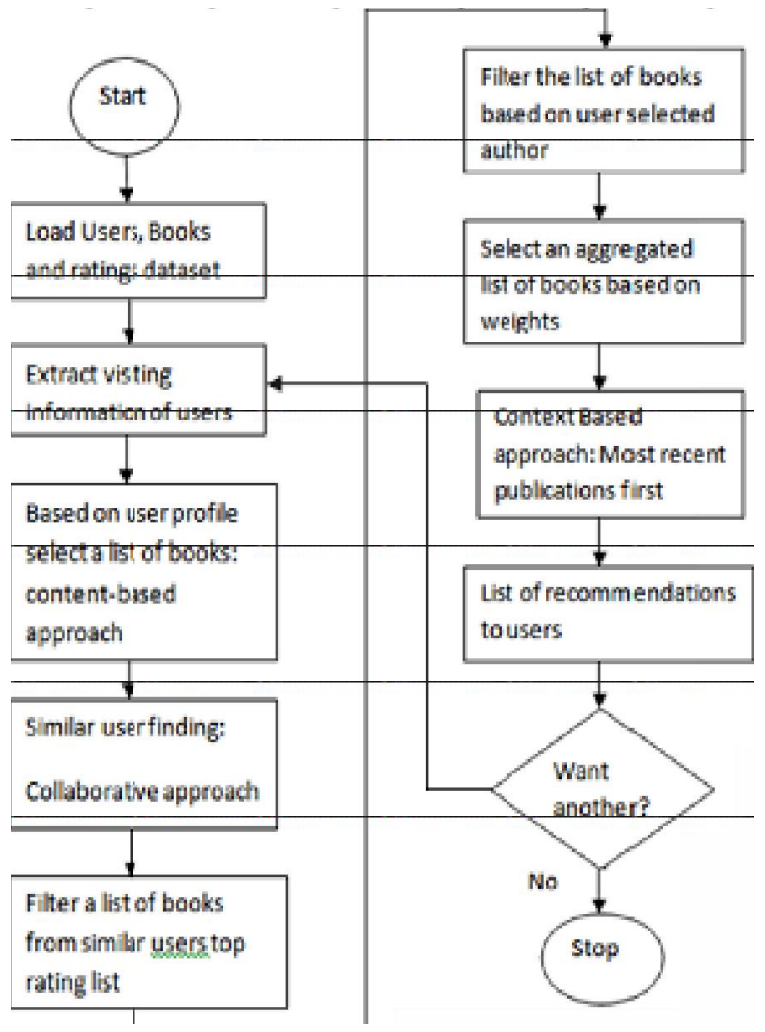

FIG. 3. FLOW CHART OF PROPOSED APPROACH
In step-7, the system combine the difference of rating calculated in step-5 with active user given rating to get the predicted rating. In step- 8 , the system calculates a weighted rating list from the predicted rating computed in step-6 and rating frequency computed in step-5. In step-9, the system finds the books of user's interest from the books dataset. This list of books is then assigned a weight $\mathrm{W}_{2}$ in step-10 (Equation (4)).

$\mathrm{J} 2_{\mathrm{i}}=\mathrm{W}_{2} * \mathrm{r}_{\mathrm{ki}}$

Here $r_{k i}$ is the rating of ith book from the list $\mathrm{K}$.

In step-11, three lists of books are combined using an aggregated function. The aggregation function finally creates a single consolidated list of books using the weights of these three lists of books. The aggregation function is computed as Equation (5):

$\mathrm{ListS}=\frac{(\mathrm{J} * 4+\mathrm{M} * 2+\mathrm{N} * 5)}{(4+2+5)}$

This aggregated list of books is filtered based on their publication year in step-12. The most recent books are recommended first followed by a filtered list of books recommended to the active user. The filtration processes and weights assignment to lists of books at different stages in our method finally provides more accurate recommendations to the users that matches their preferences in the best possible way.

The proposed algorithm of our hybrid recommendation approach is provided in Table 1.

\section{PERFORMANCE EVALUATION}

Performance of the proposed recommender system is evaluated using two methods i.e. objective and subjective

Mehran University Research Journal of Engineering \& Technology, Volume 37, No. 4, October, 2018 [p-ISSN: 0254-7821, e-ISSN: 2413-7219] 
evaluation. Objective evaluation scheme relies on computing the metrics such as precision, recall, FMeasure, etc. Whereas in subjective evaluation, performance is evaluated based on the user feedback/ rating. We have used both objective and subjective evaluation to measure the performance of the proposed hybrid recommender system. More specifically, precision, recall, accuracy, and F-Measure are used for objective evaluation, whereas, usability, user satisfaction, and relevance parameters are used to obtain the user feedback for subjective evaluation. We have used "Book Crossing" dataset for performance evaluation. The details of the dataset used and experimental results are provided in this section.

\subsection{Dataset}

A standard dataset "Book Crossing" is used to measure the performance of the proposed method. Ziegler et. al. [26] created the Book crossing dataset that contains 278,858 users providing a rating range of $1,149,780$ on 271,379 books. Book crossing dataset contains three tables namely, BX-Book.sql, BX-Books-Ratings.sql, and BX-Users.Sql.
BX-Book.sql file contains 271380 books with the information of ISBN, Book Title, Book Author, Publication Year, Publisher, Book Genre, and Image. BX-BooksRatings.sql includes the ratings of different books, user IDs of those who rated these books, and ISBN number. Users have provided the rating on a scale of 1-10 where 10 represents the highest rated book and 1 is the lowest rated book. BX-Users.sql comprises of user information of 2,76,272 users that consist of users ID, location, and age. Ziegler et. al. [26] have performed an offline as well as online analysis on the book crossing dataset. This analysis facilitated them to collect $1,157,112$ ratings and 278,858 fellows of books crossing and found that $1,157,112$ different and distinct ISBNs were referred.

\subsection{Experimental Results}

Experimental results of the proposed method are discussed in this section. An android application is specifically developed in this application based on the proposed hybrid recommendation approach and results are obtained using the book crossing dataset. The proposed application performs the search of the relevant books based on the user input query. We have provided

TABLE 1. PROPOSED HYBRID RECOMMENDATION ALGORITHM

\begin{tabular}{|c|c|c|c|c|}
\hline Number of Users & Accuracy & Precision & Recall & F-Measure \\
\hline 60 & 0.74 & 0.76 & 0.76 & 0.76 \\
\hline 80 & 0.74 & 0.76 & 0.74 & 0.75 \\
\hline 100 & 0.75 & 0.75 & 0.71 & 0.73 \\
\hline 120 & 0.74 & 0.74 & 0.7 & 0.72 \\
\hline 140 & 0.71 & 0.7 & 0.65 & 0.66 \\
\hline 160 & 0.7 & 0.69 & 0.63 & 0.64 \\
\hline 180 & 0.69 & 0.68 & 0.6 & 0.60 \\
\hline 200 & 0.65 & 0.63 & 0.57 & 0.69 \\
\hline Average & 0.72 & 0.71 & 0.67 & \\
\hline
\end{tabular}

Mehran University Research Journal of Engineering \& Technology, Volume 37, No. 4, October, 2018 [p-ISSN: 0254-7821, e-ISSN: 2413-7219] 
both the objective and subjective evaluation results of the proposed hybrid recommendation scheme. Each of the evaluation mechanism is discussed in this section.

\subsubsection{Objective Evaluation}

For objective evaluation of the proposed method, we have measured the performance on precision, recall, accuracy, and F-Measure. The above mentioned parameters are used to evaluate the proposed hybrid approach for mobile platform. An android application is specifically designed based on the proposed hybrid recommendation scheme using the book crossing dataset to recommend the relevant books based on the users interest. The proposed android application has been provided to multiple users and asked them to feed input queries at the same time to obtain the recommendations. The number of concurrent access and usage of application by multiple users have increased in each experiment (i.e. 60-200 users). In the first experiment 60 users of different gender have used the proposed recommender system at the same time. Precision, recall, F-Measure, and accuracy are computed for the 60 users collectively. Similarly, in the next experiment 80 users have used the application concurrently and the objective evaluation metrics are computed. We designed 8 different experiments in the same way by increasing the number of users by a factor of 20 in each next experiment. The recommendations provided by the proposed application to the users are listed and further used to compute the precision, recall, F-Measure, and Accuracy as shown in Table 2.

Existing commercial recommender systems usually offer a list of recommended items I (a.k.a Top-I recommendation) to each user based on his/her preference as compared to provide the predicted item ratings to the user. Precision and recall evaluation measures are frequently employed by existing viable recommender systems. We also used these measures for objective performance evaluation. The details of objective metrics computation are discussed here.

For recall metric computation against each user $\mathrm{U}$, books are ranked in the proposed scheme based on the predicted rating $\mathrm{S}$. If the user find any book interesting or attractive then the corresponding book is marked as relevant to the user. In our case, recall is defined as the fraction of relevant books that are included in the Top-I ranked list of recommendations listed as $\mathrm{M}(\mathrm{I}, \mathrm{U})$ from all of the relevant books $\mathrm{M}(\mathrm{U})$ present in the dataset for user $\mathrm{U}$. Recall is computed as Equation (6):

$\mathrm{R}(\mathrm{I}, \mathrm{U})=\frac{\mathrm{M}(\mathrm{I}, \mathrm{U})}{\mathrm{M}(\mathrm{U})}$

\section{TABLE 2. OBJECTIVE EVALUATION OF THE PROPOSED} HYBRID RECOMMENDATION APPROACH

\begin{tabular}{|c|c|}
\hline (i) & Get Genre and Author name from user ' $U$ '. \\
\hline \multirow[t]{5}{*}{ (ii) } & Check if user already exists \\
\hline & If true \\
\hline & $\begin{array}{l}\text { Check items set ' } N \text { ' from user ' } U \text { ' profile that } \\
\text { user liked in past. }\end{array}$ \\
\hline & If true \\
\hline & $\begin{array}{l}\text { Filter the items set ' } \mathrm{J} 1 \text { ' in books dataset which are } \\
\text { similar to item set ' } \mathrm{N} \text { '. }\end{array}$ \\
\hline (i) & $\begin{array}{l}\text { Calculate a weighted list ' } \mathrm{J} \text { ' of books from 'J1' } \\
\text { based on user's ratings. }\end{array}$ \\
\hline (ii) & $\begin{array}{l}\text { Check other users who like the similar genre as } \\
\text { user ' } U \text { ' like }\end{array}$ \\
\hline (iii) & $\begin{array}{l}\text { Calculate rating's frequency that users gave per } \\
\text { book. }\end{array}$ \\
\hline (iv) & $\begin{array}{l}\text { Calculate average dissimilarity in ratings that } \\
\text { users gave in regard to user ' } U \text { '. }\end{array}$ \\
\hline (v) & $\begin{array}{l}\text { Calculate predicted rating by adding the average } \\
\text { variance in rating and user ' } U \text { ' provided rating. }\end{array}$ \\
\hline (vi) & $\begin{array}{l}\text { Get weighted list ' } \mathrm{J} 2 \text { ' from predicted rating and } \\
\text { rating's frequency. }\end{array}$ \\
\hline (vii) & $\begin{array}{l}\text { Filter the books ' } \mathrm{J} 3 \text { ' from books dataset according } \\
\text { to user ' } U \text { ' Author choice. }\end{array}$ \\
\hline (viii) & Calculate a weighted list of books ' $N$ ' from ' $J 3$ '. \\
\hline (ix) & $\begin{array}{l}\text { Get an aggregated weighted rating list ' } \mathrm{S} \text { ' from } \mathrm{J} \text {, } \\
\mathrm{M} \text { and } \mathrm{N} \text {. }\end{array}$ \\
\hline$(\mathrm{x})$ & $\begin{array}{l}\text { Filter the aggregated weighted list 'S' based on the } \\
\text { publication year. Most recent published books are } \\
\text { recommended first. }\end{array}$ \\
\hline (xi) & $\begin{array}{l}\text { Recommend top most weighted rating Books to } \\
\text { user ' } U \text { '. }\end{array}$ \\
\hline
\end{tabular}


High values of R(I,U) represents more accurate Top-I recommendations. For performance evaluation we have obtained the results on different ranges of user input $(60-200)$ in the proposed work. More specifically, we computed the recall based on the outcome provided by the proposed application against the multiple user inputs. In addition, the results are accumulated for multiple users in different numbers that are $60,80,100$, $120,140,160,180$, and 200 using the application at the same time. In addition, we have also computed the average of all results obtained on different number of users as Equation (7):

$\mathrm{R}_{\mathrm{avg}}(\mathrm{I}, \mathrm{U})=\sum_{\mathrm{i}=1}^{\mathrm{Z}} \frac{\mathrm{M}(\mathrm{I}, \mathrm{U})}{\mathrm{M}(\mathrm{U})}$

Where $\mathrm{R}_{\text {avg }}(\mathrm{I}, \mathrm{U})$ represents the average recall value. It can be observed from Table 2 that the proposed scheme offers better recall rates in terms of providing the topranked recommendations among all of the relevant books.

Precision metric is also applied frequently to evaluate the performance of the recommender systems. In our case, precision represents the segment of relevant books amongst the total books (labelled as I) recommended to the user U. We computed the precision as Equation (8):

$P(I, U)=\sum_{i=1}^{z} \frac{M(I, U)}{I}$

Similarly, we have computed the precision against multiple number of users range (60-200). Likewise, average recall, we have also computed the average of all results obtained on different number of users for precision as Equation (9):

$$
\mathrm{P}_{\mathrm{avg}}(\mathrm{I}, \mathrm{U})=\sum_{\mathrm{i}=1}^{\mathrm{z}} \frac{\mathrm{M}(\mathrm{I}, \mathrm{U})}{\mathrm{I}}
$$

Where $\mathrm{P}_{\text {avg }}(\mathrm{I}, \mathrm{U})$ represents the average precision value. As shown in Table 1, the proposed approach is very effective in terms of providing relevant recommendation books among the total books provided to the user.

We also computed the F-Measure as well against the recommendations offered by the proposed method. F-Measure represents the mean of precision and recall and lies in the range of $0-1$, where 1 represents the maximum accuracy of the recommendation and 0 is the lowest in our case. F-Measure is computed as Equation (10):

$$
\text { F - Measure }=\frac{2 \times(\mathrm{P}(\mathrm{I}, \mathrm{U}) \times \mathrm{R}(\mathrm{I}, \mathrm{U}))}{\mathrm{P}(\mathrm{I}, \mathrm{U})+\mathrm{R}(\mathrm{I}, \mathrm{U})}
$$

From Table 1 it can be clearly observed that the proposed hybrid recommendation system provides superior recommendation performance. The average F-Measure of 0.8 indicates the effectiveness of the proposed recommendation scheme for better accuracy in terms of relevant books recommendation to the users.

Accuracy rate of the proposed hybrid recommendation approach is also computed to indicate the effectiveness of the proposed method in terms of clearly classifying the relevant and irrelevant recommendations out of the total possible recommendations available. Accuracy rate is computed as Equation (11):

$\mathrm{A}=\frac{\mathrm{TP}+\mathrm{TN}}{\mathrm{I}}$

Where TP denotes true positive that represents the relevant recommendations, $\mathrm{TN}$ denotes true negative that

Mehran University Research Journal of Engineering \& Technology, Volume 37, No. 4, October, 2018 [p-ISSN: 0254-7821, e-ISSN: 2413-7219] 
represents the irrelevant recommendations and I represent the total books recommended to the users as described earlier. For TP, user provides the input of interests to verify the relevant recommendations. For TN, user provides any input query which lies out of bound of his/ her interests to verify whether it provides irrelevant recommendations according to his taste but relevant according to the input.

\subsubsection{Subjective Evaluation}

The proposed hybrid recommendation approach is also evaluated in a subjective way. Subjective evaluation is based on user feedback or ratings on a subjective scale provided by multiple users based on their experience. We performed the subjective evaluation on 20 test subjects of different genders of computer science domain at undergraduate level. We collected the subjective ratings of different users on three parameters namely Usability, User Satisfaction, and Relevance (in terms of recommendations). The subjective scale lies in the range of 1-10 where 1 represents the lowest rating and 10 is the highest rating. We provided the application to the users and asked them to use for a week and observe the behavior of the application in terms of providing relevant recommendations, ease of use, and user satisfaction. The above mentioned subjective parameters are chosen to obtain the feedback of users from multiple aspects that covers both the functional and non-functional aspect of the application. Relevance parameter is selected to provide the user feedback in terms of providing accurate recommendations to the users. Usability parameter is chosen to obtain the user feedback from multiple aspects such as ease of use, efficiency, and effort required to perform certain tasks. User Satisfaction parameter is selected to obtain the user feedback in terms of overall perception about the application and satisfaction regarding the functional and non-functional capabilities of the proposed system. Subjective evaluation ratings of the 20 test subjects against the three above mentioned selected parameters are provided in Table 3 . The average scores of for Relevance, Usability, and User Satisfaction signify the effectiveness of the proposed hybrid recommendation scheme in terms of meeting users' requirements from both functional and non-functional aspects.

\subsubsection{Performance Comparison}

We designed an experiment to compare the performance of the proposed method against existing state-of-the-art recommender systems [21] including collaborative and content based approaches. We have implemented both collaborative and content based approaches independently and obtained the results of these approaches. Collaborative filtering is implemented using the user's profile information, whereas, content based approach is developed using those books rated high by most of the users in the neighborhood. A comparative analysis is performed using precision, recall, F-Measure, and Accuracy. The reported results of the existing approaches and the proposed hybrid recommendation scheme is provided in Figs. 4-7. As it can be observed from Figs. 4-7 that the proposed hybrid recommendation approach outperforms the comparative methods in terms of precision, recall, accuracy and F-Measure.

The comparative results of precision metric are provided in Fig. 4. As shown in Fig. 4 that the proposed recommender system outperforms the comparative methods in terms of providing relevant recommendation books among the total items provided to the user. Precision on y-axis represents the average range between 0 and 1 .

Similarly, the comparative results of accuracy metric are presented in Fig. 5. It can be observed from Fig. 5 that the proposed system provides better accuracy as compared to the existing recommender systems.Accuracy on y-axis represents the average range between 0 and 1 .

Mehran University Research Journal of Engineering \& Technology, Volume 37, No. 4, October, 2018 [p-ISSN: 0254-7821, e-ISSN: 2413-7219] 
The recall evaluation results are reported in Fig. 6 and it can be concluded after analyzing Fig. 6 that the proposed hybrid scheme generates better recall rates, in terms of providing the top-ranked recommendations among all of the relevant books, as compared to existing recommender systems.

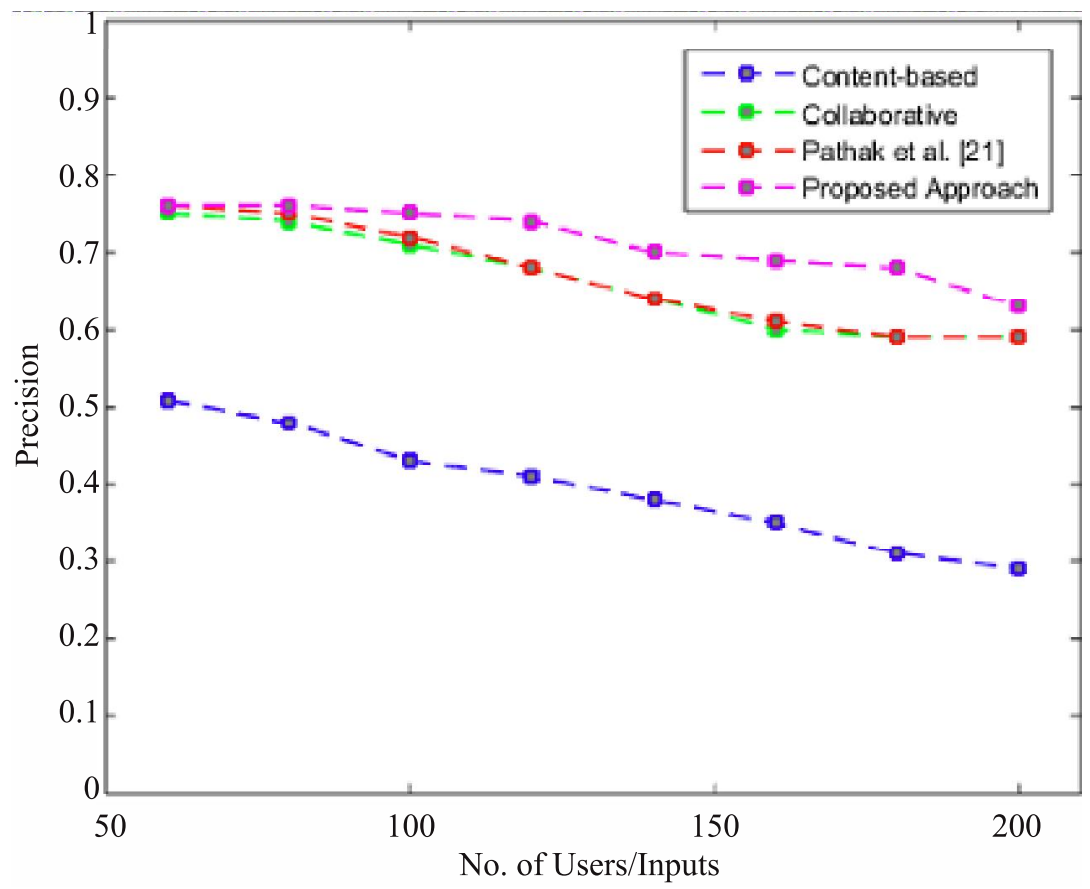

FIG. 4. COMPARATIVE ANALYSIS USING PRECISION AS EVALUATING PARAMETER

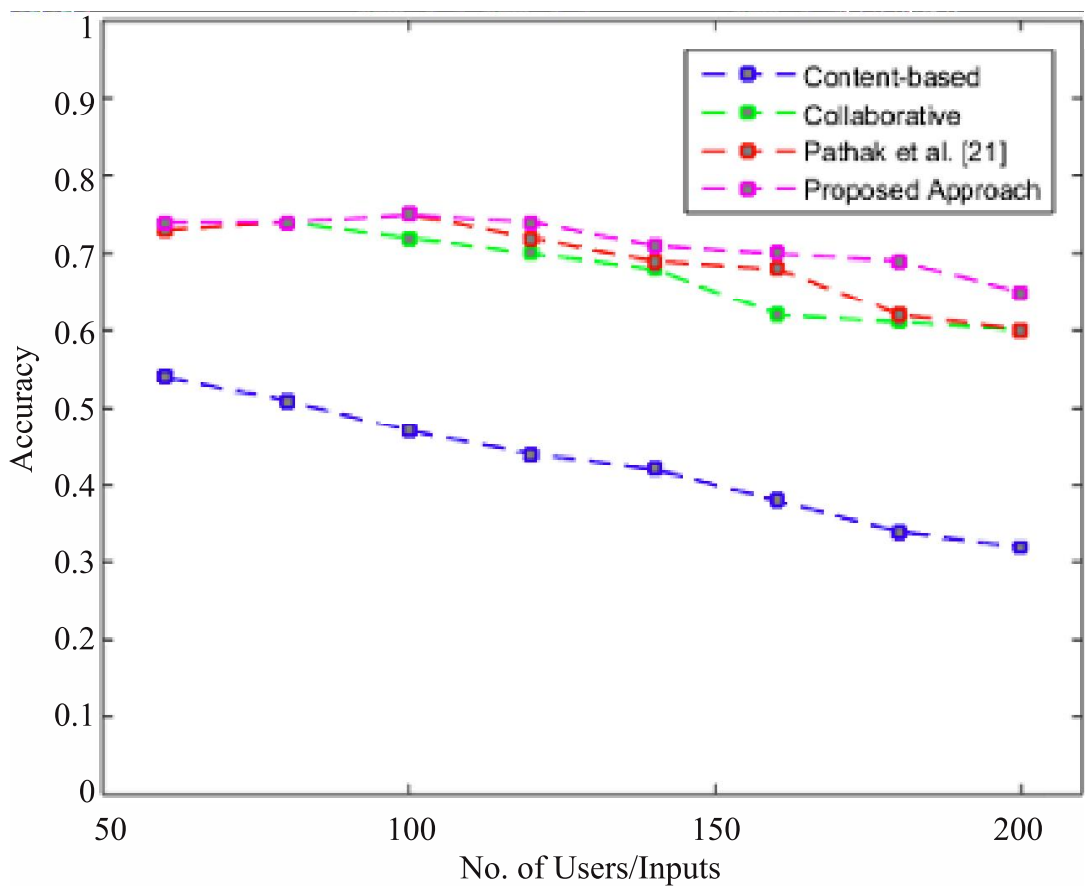

FIG. 5. COMPARATIVE ANALYSIS USING ACCURACY AS EVALUATING PARAMETER

Mehran University Research Journal of Engineering \& Technology, Volume 37, No. 4, October, 2018 [p-ISSN: 0254-7821, e-ISSN: 2413-7219] 
Recall on $y$-axis represents the average range between 0 and 1.

Likewise, F-Measure evaluation results are mentioned in

Fig. 7 which shows the effectiveness of the proposed method in terms of recommending the top-ranked relevant books to the users based on their interests. F-Measure on y-axis represents the mean of precision and recall and lies in the range of $0-1$

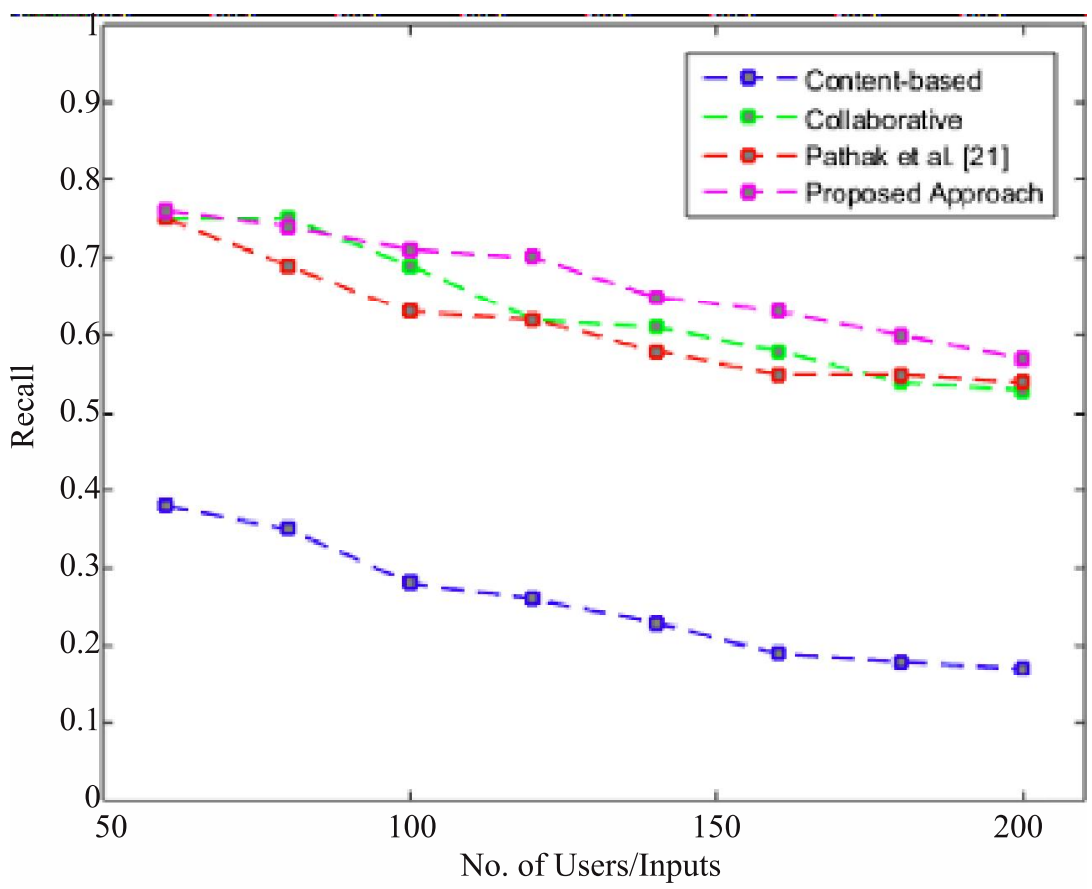

FIG. 6. COMPARATIVE ANALYSIS USING RECALL AS EVALUATING PARAMETER

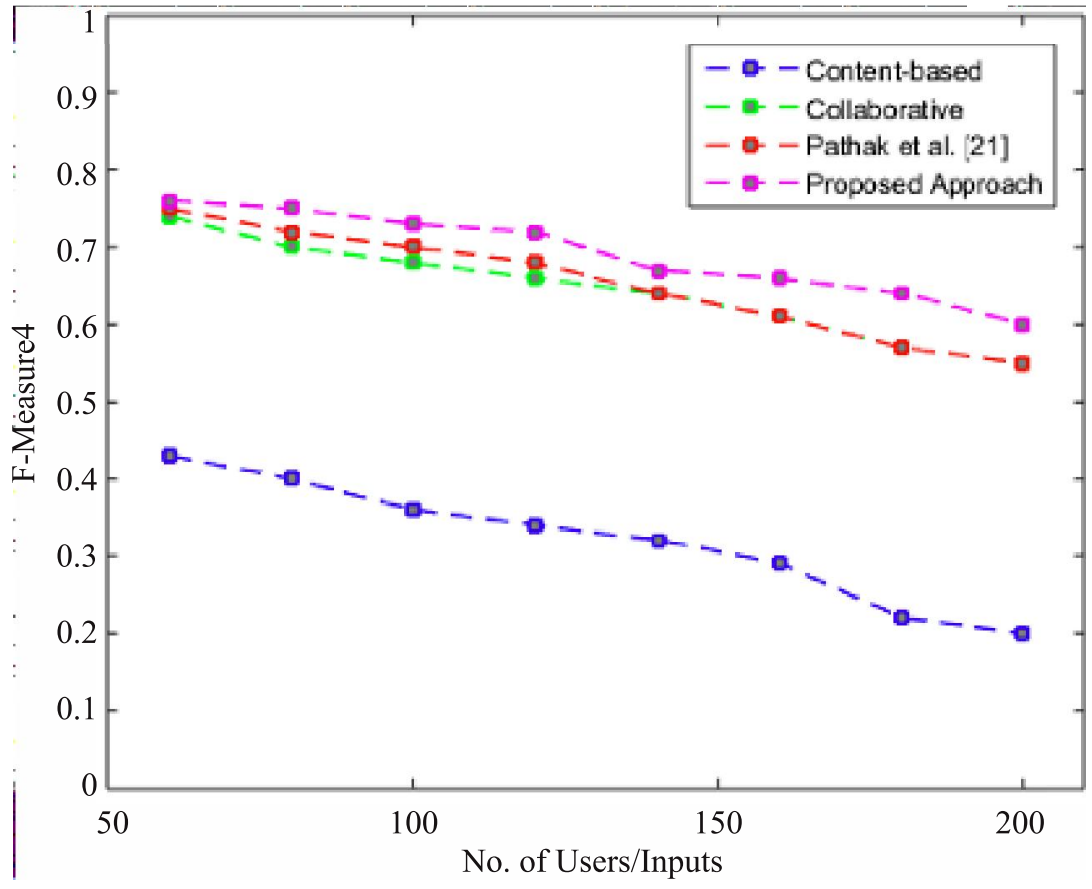

FIG. 7. COMPARATIVE ANALYSIS USING F-MEASURE

Mehran University Research Journal of Engineering \& Technology, Volume 37, No. 4, October, 2018 [p-ISSN: 0254-7821, e-ISSN: 2413-7219] 
More specifically, content based approach provides an average precision, recall, F-Measure, and accuracy $0.39,0.27,0.32$, and 0.42 of respectively. Collaborative filtering approach achieves an average precision, recall, F-Measure, and accuracy of $0.66,0.62,0.64$, and 0.68 respectively. Similarly, NOVA hybrid approach discussed in [21] provides an average precision, recall, F-Measure, and accuracy of $0.67,0.63,0.65$, and 0.69 respectively. The proposed hybrid books recommendation scheme achieves an average precision, recall, F-Measure, and accuracy of $0.71,0.67$, 0.69 , and 0.72 respectively. The comparative analysis of results signify that the proposed hybrid recommendation approach outperforms the comparative approaches in terms of recommendation.

\section{CONCLUSION}

In this paper we proposed an efficient hybrid books recommender system for mobile platform. The proposed hybrid scheme adopts the traits of content based and collaborative filtering approaches in addition of the context based approach to facilitate the user in terms of recommending the most relevant and latest published books. We have developed an android application based on the proposed recommendation approach. Book crossing dataset is used for performance evaluation. We have tested the proposed android based recommender system using objective and subjective evaluation parameters. Experiments have exposed that overall recommendation of proposed algorithm is improved using user's favorite author. Moreover, it has been observed that using different weights to different lists of filtered books achieve better recommendations. A comparative analysis of the proposed hybrid recommender system is also performed against the existing recommender systems which shows that the proposed system provides superior recommendation performance as compared to existing systems. The proposed hybrid recommendation scheme can easily be extended for other domains in the future.

\section{ACKNOWLEDGEMENT}

This research work is supported by University of Engineering \& Technology, Taxila, University of Gujrat, and International Islamic University, Islamabad, Pakistan. The authors are thankful to all these institutions for providing the required resources to perform this research.

\section{REFERENCES}

[1] Xiwang, Y., Guo, Y., Liu, Y., and Steck, H., "A Survey of Collaborative Filtering Based Social Recommender Systems", Computer Communications, Volume 41, pp. 1-10, 2014.

[2] deGemmis, M.,, Lops, P., Musto, C., Narducci, F., and Semeraro, G., "Semantics-Aware Content-Based Recommender Systems", Recommender Systems Handbook, pp. 119-159, Springer, USA, 2015.

[3] Ehsan, A., Radmanesh, M., and Jalili, M., "Hybrid Recommender Systems Based on Content Feature Relationship", IEEE Transactions on Industrial Informatics, 2016.

[4] Zhu, Z., and Wang, J.Y., "Book Recommendation Service by Improved Association Rule Mining Algorithm”, IEEE International Conference on Machine Learning and Cybernetics, 2007. 
[5] Rana, C., and Jain, S.K., "Building a Book Recommender System Using Time Based Content Filtering”, WSEAS Transactions on Computers, Volume 11, No 2, pp. 27-33, 2012.

Resnick, P.,Iacovou, N., Suchak, M., Bergstrom, P., and Riedl, J., "GroupLens: An Open Architecture for Collaborative Filtering of Netnews", Proceedings of ACM Conference on Computer Supported Cooperative Work, 1994.

Resnick, P., and Varian, H.R., "Recommender Systems", Communications of the ACM, Volume 40, No 3, pp. $56-58,1997$

[8] Swearingen, K., and Sinha, R., "Interaction Design for Recommender Systems”, Designing Interactive Systems, Volume 6, No. 12, pp. 312-334, 2002.

DeZelar-Tiedman, C., "Doing the Library Thing ${ }^{\mathrm{TM}}$ in an Academic Library Catalog", Proceeding of International Conference on Dublin Core and Metadata Applications, pp. 211-213, 2008.

[10] Melville, P.. and Sindhwani. V., "Recommender Systems", Encyclopedia of Machine Learning, Volume 1, No. 338, pp. 829-838, Springer, 2010.

[11] Breese, J.S., Heckerman, D., and Kadie, C., "Empirical Analysis of Predictive Algorithms for Collaborative Filtering", Proceedings of $14^{\text {th }}$ Conference on Uncertainty in Artificial Intelligence, Morgan Kaufmann Publishers Inc, 1998.

[12] Linden, G., Smith, B., and York, J., "Amazon.com Recommendations: Item-to-Item Collaborative Filtering", IEEE Internet Computing, Volume 7, No. 1, pp. 76-80, 2003.

[13] Sarwar, B., Karypiset, G., Konstan, J., and Riedl, J., "ItemBased Collaborative Filtering Recommendation Algorithms", Proceedings of 10th ACM International Conference on World Wide Web, 2001.
[14] Koren, Y., Bell, R., and Volinsky, C., "Matrix Factorization Techniques for Recommender Systems", Computer, Volume 42, No. 8, pp. 30-37, 2009.

[15] Mooney, R.J., and Roy, L., "Content-Based Book Recommending Using Learning for Text Categorization", Proceedings of ACM $5^{\text {th }}$ Conference on Digital libraries, 2000.

[16] Melville, P., Mooney, R.J., and Nagarajan, R,. "ContentBoosted Collaborative Filtering for Improved Recommendations", Proceedings of $18^{\text {th }}$ National Conference on Artificial Intelligence, pp. 187-192, Edmonton, Canada, July, 2002.

[17] Kim, D., Park, C., Oh, J., and Yu, H., "Deep Hybrid Recommender Systems via Exploiting Document Context and Statistics of Items", Information Sciences, Volume 417, pp. 72-87, 2017.

[18] Pazzani, M., and Billsus, D., "Learning and Revising User Profiles: The Identification of Interesting Web Sites", Machine learning, Volume 27, No. 3, pp. 313-331, 1997.

[19] Pera, M.S., and Ng, Y.K., "With a Little Help from My Friends: Generating Personalized Book Recommendations Using Data Extracted from a Social Website", Proceedings of IEEE/WIC/ACM International Conferences on Web Intelligence and Intelligent Agent Technology, Computer Society, Volume 1, pp. 96-99, 2011.

[20] Ristoski, P., Mencía. E.L., and Paulheim, H., “A Hybrid Multi-Strategy Recommender System Using Linked Open Data”, Semantic Web Evaluation Challenge, Volume 475, No. 6, pp. 150-156, Springer, 2014.

[21] Pathak, D., Matharia, S., and Murthy, C., "NOVA: Hybrid Book Recommendation Engine", IEEE 3rd International Conference on Advance Computing, 2013.

[22] Smyth, B., and Cotter, P., "Personalized Electronic Program Guides for Digital TV", AI Magazine, Volume 22, No 2, pp. 89, 2001. 
[23] Tewari, A.S., Kumar, A., and Barman, A.G., "Book Recommendation System Based on Combine Features of Content Based Filtering, Collaborative Filtering and Association Rule Mining”, IEEE International Advance Computing Conference, 2014.

[24] Sohail, S.S., Siddiqui, J., and Ali, R., "Book Recommendation System Using Opinion Mining Technique", IEEE International Advances in Computing, Communications and Informatics, 2013.
[25] Sase, A., Varunet, K., Rathod, S., and Patil, D., "A Proposed Book Recommender System”, International Journal of Advanced Research in Computer and Communication Engineering, Volume 4, No. 2, pp. 481-483 2015 .

[26] Ziegler, C.-N., McNee, S.M., Konstan, J.A., and Lausen, G., "Improving Recommendation Lists Through Topic Diversification", Proceedings of $14^{\text {th }}$ ACM International Conference on World Wide Web, 2005. 